The Soft-SixthFinger: a wearable EMG controlled robotic extra-finger for grasp compensation in chronic stroke patients

This is the peer reviewed version of the following article:

Original:

Hussain, I., Salvietti, G., Spagnoletti, G., Prattichizzo, D. (2016). The Soft-SixthFinger: a wearable EMG controlled robotic extra-finger for grasp compensation in chronic stroke patients. IEEE ROBOTICS AND AUTOMATION LETTERS, 1(2), 1000-1006 [10.1109/LRA.2016.2530793].

Availability:

This version is availablehttp://hdl.handle.net/11365/992084

since 2019-05-07T18:30:12Z

Published:

DOI:10.1109/LRA.2016.2530793

Terms of use:

Open Access

The terms and conditions for the reuse of this version of the manuscript are specified in the publishing policy. Works made available under a Creative Commons license can be used according to the terms and conditions of said license.

For all terms of use and more information see the publisher's website.

(Article begins on next page) 


\title{
The Soft-SixthFinger: a Wearable EMG Controlled Robotic Extra-Finger for Grasp Compensation in Chronic Stroke Patients
}

\author{
Irfan Hussain $^{1}$, Gionata Salvietti ${ }^{1}$, Giovanni Spagnoletti ${ }^{1}$, and Domenico Prattichizzo ${ }^{1,2}$
}

\begin{abstract}
This paper presents the Soft-SixthFinger, a wearable robotic extra-finger designed to be used by chronic stroke patients to compensate for the missing hand function of their paretic limb. The extra-finger is an underactuated modular structure worn on the paretic forearm by means of an elastic band. The device and the paretic hand/arm act like the two parts of a gripper working together to hold an object. The patient can control the flexion/extension of the robotic finger through the eCap, an Electromyography (EMG) interface embedded in a cap. The user can control the device contracting the frontalis muscle by moving his or her eyebrows upwards. The Soft-SixthFinger has been designed as tool that can be used by chronic stroke patients to compensate for grasping in many Activities of Daily Living (ADL). It can be wrapped around the wrist and worn as a bracelet when not used. The light weight and the complete wireless connection with the EMG interface guarantee a high portability and wearability. We tested the device with qualitative experiments involving six chronic stroke patients. Results show that the proposed system significantly improves the performance of the considered tests and the autonomy in ADL.
\end{abstract}

\section{INTRODUCTION}

The number of stroke patients with long-term disabilities of the upper limb is of the order of millions [1]. Findings of available prospective cohort studies indicate that $33 \%$ to $66 \%$ of the stroke patients show no recovery of upper limb functions after six months from the stroke [2], whereas only $5 \%$ to $20 \%$ demonstrate complete functional recovery [3]. Several robotic devices have been developed to provide safe, intensive rehabilitation to patients with mild to severe motor impairments after neurologic injury [4], [5]. The use of robotic devices in rehabilitation can provide high-intensity, repetitive, task-specific, interactive treatment of the impaired upper limb.

The majority of the robotic devices, however, are designed to increase the functional recovery of the hand/arm in the first months after stroke, when, in some cases, biological restoring and plastic reorganization of the central nervous system take place. To the best of our knowledge, few devices have been specifically designed as robotic tools to compensate for hand/arm function when in the paretic upper limb the motor deficit is stabilized [6], [7].

In tools designed for actively compensate the hand grasping function, the wearability of the robot becomes a key feature in the design process, since the tools should be

This work was supported in part by the EU FP7/2007-2013 project no. 601165 WEARHAP and in part by the EU Horizon 2020/2015, project no. 688857 SOFTPRO.

${ }^{1}$ Università degli Studi di Siena, Dipartimento di Ingegneria dell'Informazione, Via Roma 56, 53100 Siena, Italy. \{hussain, salviettigio, prattichizzo\}@dii.unisi.it

${ }^{2}$ Department of Advanced Robotics, Istituto Italiano di Tecnologia, Via Morego 30, 16163 Genoa, Italy.

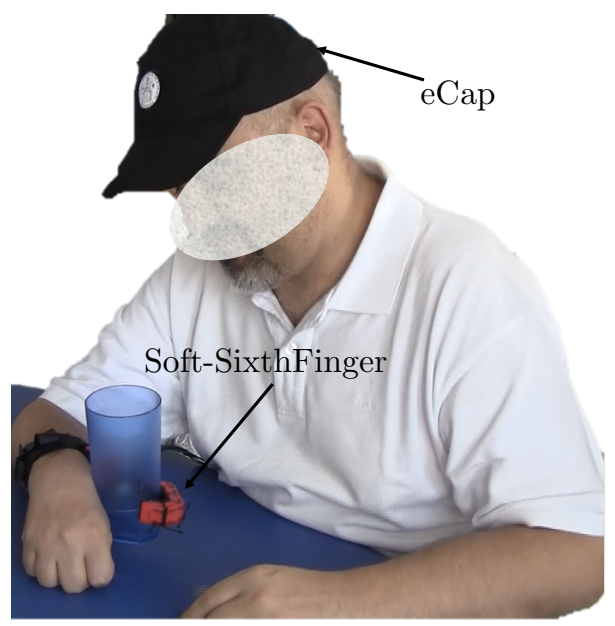

Fig. 1. The Soft-SixthFinger. The robotic extra-finger works with the paretic limb to compensate for hand grasp functionality. The patient can control the device thanks to a wearable EMG interface embedded in a cap called eCap.

used by the patients also outside the rehab facilities. In [8], Pons et al. outlined two possible categories of wearable robots: exoskeletons and prosthetic robots. The former are designed to complement the ability of the human limb and restore the handicapped function usually mapping onto the anatomy of the human limb. The latter are electro-mechanical devices that substitutes for lost limbs after amputation.

In this work, we enrich these definitions by introducing a wearable robot which is grounded on the human body, but that is not mechanically coupled with the human limb. We do not attempt to assist the paretic hand/arm motion of the patient, but rather we add just what is needed to grasp: an extra thumb. Referring to the scenario in Fig. 1, the robotic extra-finger is worn on the user forearm and can accomplish a given task in cooperation with the paretic limb. A preliminary prototype, called the Robotic Sixth Finger, has been proposed in [9], [10], [11]. The fully actuated structure allows the Robotic Sixth Finger to actively shape around an object, but the resulting size and weight affect the device portability and wearability. The patient can regulate the finger flexion/extension through a wearable switch embedded in a ring worn on the healthy hand. The ring interface dramatically simplifies the interaction with the device, although, when performing bimanual tasks or when grasping an object, the subject has to be careful not to activate the switch unintentionally.

The Soft-SixthFinger presented in this paper shares the working principle with the previous prototypes. The hand 
grasping function is compensated by means of the extrafinger opposition to the paretic limb as shown in Fig. 1. The Soft-SixthFinger has been designed to guarantee high wearability and portability. The device can be worn as a bracelet when it is not used. The patient can switch from the bracelet to the operative position by using his or her healthy hand. Moreover, in the previous versions of the device, the batteries and all the electronic boards for the control were worn in the paretic arm by means of an elastic band. The arising weight limited the mobility on the patient's paretic arm. In the Soft-SixthFinger, we moved all the batteries and the electronics board from the paretic arm to a box that can be fixed in the user belt. The result is a sensible reduction of the weight the user has to support with the paretic arm.

The Soft-SixthFinger kinematics and actuation are inspired by recent works on underactuated compliant robotic hands [12], [13], [14]. In particular, the robotic extra-finger is passively compliant thanks to its flexible joints. Only one motor is used to regulate the device flexion through a tendon-driven actuation. The advantage of this solution with respect to the previous versions of the device are threefold. The compliance in the structure simplifies the control of the device that can passively adapt to the shape of the grasped object [12]. Flexible joints increase also the robustness against undesired contacts with the environment [15]. Finally, the tendon-driven underactuation reduces the total weight of the device increasing its portability and wearability.

In this paper, we also present a new user control interface for the Soft-SixthFinger called eCap. Such interface can recognize, through the acquisition of the Electromyography (EMG) signal read at the frontalis muscle of the patient, when the patient voluntary moves his or her eyebrows upwards. Frontalis muscle contractions generate events that switches the states of a Finite State Machine (FSM) which regulates the finger flexion/extension. The whole system is embedded in a cap. The patients can wear the interface using only their healthy hand. Electrodes can be easily placed on the patient's forehead just wearing the interface. The eCap maintains the simplicity of a switch, but leaves the patient free to use his or her healthy hand.

To test the proposed device for grasp compensation, we set up two experiments involving six subjects in a chronic state. Firstly, the subjects were asked to perform the tasks included in the Frenchay Arm Test [16]. This test includes different manipulation tasks that patients were not able to perform, but were successfully accomplished with the aid of the robotic extra-finger. In particular, all the patients gained two points in the five points scale considered in the test. Although this test is usually adopted to evaluate the rehabilitation progress without the help of compensation tools, we wanted to verify the performance improvement in a standard test involving grasping tasks. In addition, we asked the patients to perform common bimanual tasks including opening cans and jars with different closing system and shapes to verify if the device can solve common issues in the execution of ADL.

The paper is organized as follows. In Section II the design of the Soft-SixthFinger is explained in detail. Section III deals with the EMG interface used to control the device flexion/extension. In Section IV the results of the tests executed by chronic stoke patients are reported, while in
TABLE I

THE SOFT-SIXTHINGER TECHNICAL DETAILS

\begin{tabular}{|l|r|}
\hline Module dimensions & $2.0 \times 3.1 \times 1.2 \mathrm{~cm}^{3}$ \\
\hline Module dimensions when connected & $2.0 \times 2.2 \times 1.2 \mathrm{~cm}^{3}$ \\
\hline Support base dimensions & $6.4 \times 7.0 \times 0.35 \mathrm{~cm}^{3}$ \\
\hline Module weight & $3.6 \mathrm{~g}$ \\
\hline Total weight: finger + support base & $140 \mathrm{~g}$ \\
\hline \hline Actuator control unit box dimensions & $7.1 \times 7.1 \times 4.5 \mathrm{~cm}^{3}$ \\
\hline Actuator control unit box weight & $146 \mathrm{~g}$ \\
\hline Actuator max torque & $1 \mathrm{Nm} @ 7.5 \mathrm{~V}$ \\
\hline Max. current required & $900 \mathrm{~mA}$ \\
\hline Max. operating angles & $684 \mathrm{deg} / \mathrm{sec}$ \\
\hline Max. non-loaded velocity & $7.5 \mathrm{~V}, 2.2 \mathrm{Ah}$ \\
\hline Battery & $7.4 \mathrm{~N}$ \\
\hline \hline Max. Force at fingertip & $1.4 \mathrm{~kg}$ \\
\hline Max. payload & deg, endless turn \\
\hline
\end{tabular}

Section V conclusion and future work are outlined.

\section{The Soft-SixthFinger}

\section{A. Finger structure and actuation}

The Soft-SixthFinger has been designed to be wearable, robust and capable to adapt to different object shapes. The design process has been inspired by the design of underactuated robotic hands [17]. Underactuated hands have desirable adaptability to shapes, and can be effectively implemented using relatively simple differential and elastic elements. The transmission solutions allow motion of other joints to continue after contact occurs on a coupled link, allowing the hands to passively adapt to the object shape [18].

The proposed device consists of two main parts, a flexible finger and a support base, see Fig. 2. The flexible finger has a modular structure. Each module has a 3D printed ABS (Acrylonitrile Butadiene Styrene, ABSPlus, Stratasys, USA) polymeric part that acts as a rigid link and a 3D printed thermoplastic polyurethane (Lulzbot, USA) part that realizes the flexible joint. Soft rubber pads are glued to the rigid links to increase the friction at possible contact areas. The CAD of the module is reported on the left side of Fig. 2, while the technical details are collected in Table I. The modules are connected by sliding the thermoplastic polyurethane part in the ABS part, see Fig. 2.

The Soft-SixthFinger has a single actuator used to move the whole finger through a tendon. A hole in the rigid link allows the passage of the cable (polyethylene dyneema fiber, Japan) which is used to realize the tendon driven actuation. The tendon wire runs through the finger and is attached on one side to the fingertip and on the other to a pulley rigidly connected to the actuator shaft. Due to flexible parts in the modules, the device is passively compliant. Reasons for adding passive elements are manifold, including avoiding tendon slackness and ensuring the uniqueness of the position [14]. The built-in compliant nature of the extrafinger increases its ability to grasp different objects. Shape adaptation increases the grasp performance by compensating the uncertainties in sensing, actuation and helps in stabilizing the grasp [19]. 


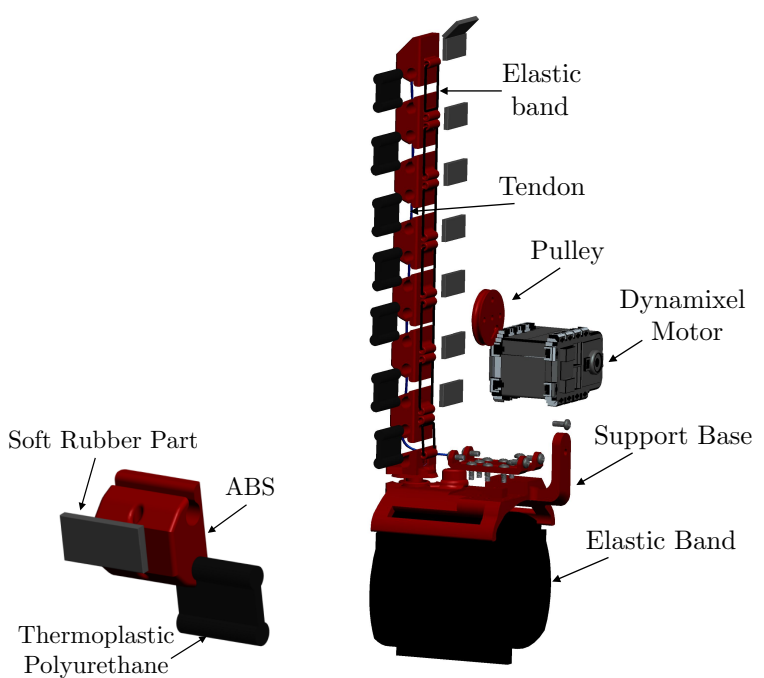

Fig. 2. The CAD exploded view of Soft-SixthFinger. On the left a single module. On the right, the structure of the device and its actuator.

The support base of the finger is realized in ABS. It contains the actuator and an elastic band that allows the user to wear the device on the forearm. The passive rotatable locking mechanism that connects the support base and the flexible finger is described in detail in Section II-B. The structure of the support base is symmetrical, feature that enables the robotic finger to be worn on both the left or right arm of the patients without any modification in the device. The actuator used is a Dynamixel servo AX12-A (Robotis, South Korea). Principal details on the motor features are reported in Table I, while for a complete description, the reader is referred to [20]. We use ArbotiX-M Robocontroller [21] to drive the Dynamixel motor. This control solution for Dynamixel motors incorporates an AVR microcontroller, Xbee wireless radio and the motor driver.

The extra-finger flexion and extension are commanded through the EMG interface described in Section III. When flexion command is selected, a desired position (300 deg, which means the extra-finger completely flexed) for the servomotor and a desired velocity (33.3 deg/s) are set. When extension is selected, the desired position is set to $0 \mathrm{deg}$, which results in the finger completely extended, with the same desired velocity.

The maximum fingertip force and the maximum payload of the device have been measured by using a dynamometer (Vernier, USA). In order to evaluate the maximum fingertip force, the device has been grounded on a table with the finger perpendicular to the table surface. The initial configuration of the finger was fully extended and it was commanded to close at the maximum torque. The dynamometer measured the force in the vertical direction and its hook was rigidly coupled with the fingertip of the Soft-SixthFinger. The constant applied force value at fingertip is presented in Table I. To evaluate the maximum payload an operator worn the SoftSixthFinger on the forearm as in Fig. 1. The operator's arm was stabilized on a table while grasping a cylindrical object (diameter $=5.7 \mathrm{~cm}$, weight $=0.5 \mathrm{~kg}$ ) with the aid of the SoftSixthFinger at its maximum torque. The grasped object was rigidly connected to the hook of dynamometer and it was

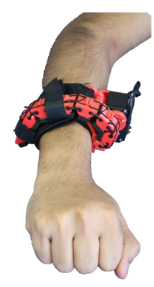

(a)

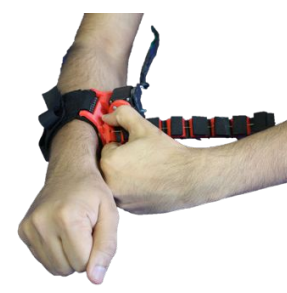

(b)

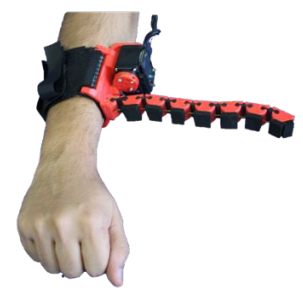

(c)
Fig. 3. From rest to working position. (a) The Soft-SixthFinger is worn as a bracelet in its rest position. (b) The patient can use his or her healthy hand to rotate the robotic finger. (c) The Soft-SixthFinger in its working position.

pulled down using the dynamometer's utility handle. The maximum pulling force was recorded as soon as the object started to slip.

\section{B. Wearability and positioning of the device}

The Soft-SixthFinger is a wearable tool that can be used by the patients in ADL where it is necessary to grasp an object. The weight of the device plays a key role in the exploitation of the residual mobility on user's paretic arm. The ArbotiX controller along with Xbee module and battery are enclosed in a 3D printed box that can be worn on the patient's belt. This solution allows to reduce the weight on the patient forearm, although it requires a cable to run from the box to the device.

The finger can be worn by the user by means of an elastic band. While not used by the patient, the extra-finger can be wrapped up on the arm as a bracelet so to reduce the encumbrance of the device. The patient can use his or her healthy hand to switch from the rest to the working position, as shown in Fig. 3. The switching between the two positions is achieved through a passive rotatable locking mechanism. This mechanism consists of two parts: one is embedded inside the support base and the other is contained in the finger starting module. A pin joint has been added in the center of both parts to allow only rotation while constraining the decoupling of both parts without unscrewing the pin joint. The mechanism has two locking positions. The locking positions are set at the working and rest positions of the extra-finger.

Together with wearability, the position of the device on the forearm has a critical role in the performance of task execution. The location of the device depends on the patient conditions and on the residual mobility of the arm/hand. The Soft-SixthFinger can be worn on the distal part of the forearm (near or on the wrist), so to obtain the grasp by opposing the device to the paretic hand. However, the distal position of the robotic finger may fail when the post stroke motor deficit is so advanced that a pathological synergism in flexion has taken place. In this case, the wrist and fingers are too much flexed not allowing successful grasping. When this pathological condition occurs, the extra-finger may be positioned more proximal at the forearm, so to let the grasp be achieved by the extra-finger opposition to the radial part of the wrist. This flexibility in the positioning is achieved thanks to the symmetrical structure and the ergonomics of the support base. The support base of the finger can be translated 


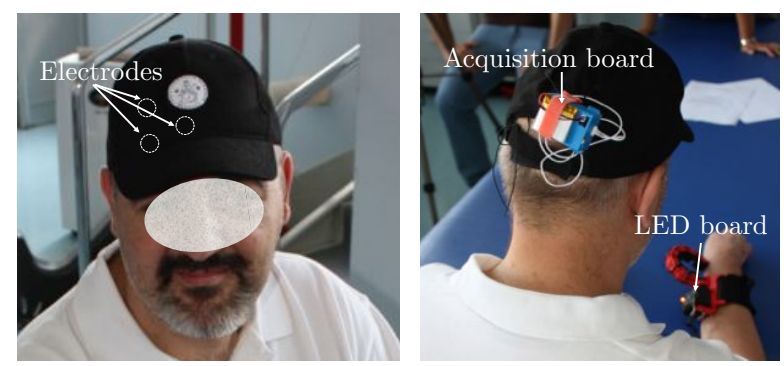

Fig. 4. The eCap interface. The EMG electrodes are placed inside the cap at front side to be positioned on the patient's forehead. The acquisition board is placed in a box on the back of the cap.

or rotated along the arm to place the finger on a suitable orientation.

\section{THE ECAP EMG INTERFACE}

The interface between the patient and the Soft-SixthFinger must be intuitive and simple. The ease of use represents an important feature, taking into consideration that a proportion of stroke patients may also complain of some cognitive deficits, possibly limiting their compliance during a demanding learning phase. In [10], [11], we presented a preliminary interface where the patient could control the robotic extrafinger motion through a switch embedded on a ring worn on the healthy hand. However, involving the healthy hand in the control of the extra-finger can interfere when performing bimanual tasks or when grasping an object, since the patient has to be careful not to activate the switch unintentionally.

In this paper, we introduce the eCap interface. The eCap is an EMG interface which maintains the principle of simplicity of the switch, but leaves the patient free to use his or her healthy hand. The electrodes and the relative electronic board for the EMG acquisition are embedded in a cap, see Fig. 4. This solution allows the patient to wear the interface using only his or her healthy hand. The EMG signals have been already successfully used to control robotic hand prosthesis [22], [23] and exoskeletons [24], [25]. The electrodes are usually placed either in the muscles coupled with the robot (exoskeleton) or in muscles where amputees still have the phantom of functions and hence they are able to generate a repeatable EMG pattern corresponding to each of the functions (prosthesis).

In the proposed interface, patients can consciously control the Soft-SixthFinger by contracting the frontalis muscle [26]. The frontalis muscle, due to a bilateral cortical representation, is always spared in case of a motor stroke, either of the left or of the right hemisphere. Activation of the muscle is achieved by moving the eyebrows upwards ${ }^{1}$. We used surface EMG electrodes to measure electrical signals associated with the patient's frontalis muscle. In particular, on the inner side of the cap, we installed silver/silver-chloride electrodes, as they present the lowest noise interface and are recommended for biopotentials recording [27]. In order to improve the quality of the EMG signal detection, the proposed system embeds three electrodes. Two are connected

\footnotetext{
${ }^{1}$ The reviewers are encouraged to watch the video enclosed with the submission.
}

TABLE II

SPECIFICATIONS OF EMG ACQUISITION BOARD

\begin{tabular}{|l|c|}
\hline EMG acquisition box dimensions & $3.5 \times 3.1 \times 4.5 \mathrm{~cm}^{3}$ \\
\hline EMG acquisition box weight & $46 \mathrm{~g}$ \\
\hline Principle & Differential voltage \\
\hline Number of electrodes & 3 \\
\hline Bandwidth & $10-400 \mathrm{~Hz}$ \\
\hline Variable Gain & $1200-4300$ \\
\hline Input Impedance & $100 \mathrm{GOhm}$ \\
\hline Common mode rejection ratio (CMRR) & $110 \mathrm{~dB}$ \\
\hline Operating voltage & $V c c=3.3 \mathrm{~V}$ \\
\hline
\end{tabular}

to the inputs of an instrumentation amplifier, the other one called "reference electrode" is connected to a mid-supply reference voltage $(1.65 \mathrm{~V})$. This technique is used to increase the common mode rejection ratio (CMRR) of the biopotential amplifier [28].

The EMG signal detected from the electrodes is acquired through a customized EMG acquisition board whose specifications are reported in Table II. The wireless communication is realized by a pair of Xbee modules ( $1 \mathrm{~mW}$ Wire Antenna). The transmitter is placed on the eCap and the receiver is located on the ArbotiX-M Robocontroller. Fig. 5 shows the wireless EMG interface with the Soft-SixthFinger. Both EMG acquisition board and XBee transmitter module are enclosed in a box and fixed on the back side of the cap, see right side of Fig. 4. The Arbotix-M controller processes the received EMG data and generates a trigger event when the signal exceeds a predefined threshold. The threshold value is evaluated for each patient during the training phase as a percentage of the maximum voluntary contraction of the frontalis muscle. It is possible to tune the sensitivity of the system acting on the potentiometer embedded in the EMG acquisition board both to filter undesired device activation due to involuntary contraction of the frontalis muscle and to set a level that is repeatable and sustainable for the user.

We have defined a high level control strategy based on a Finite State Machine (FSM) [29]. The outputs of the FSM are predefined commands based on sequences of input signals. We consider a finite number of states, transition between those states, and commands. States represent predefined motion commands for the Soft-SixthFinger, and transition roles are associated with contractions of the frontalis muscle. With one muscle contraction the patient controls the motion/stop of the finger. When the finger is stopped, two contractions in a time window of $1 \mathrm{~s}$ switch the motion direction from flexion to extension and viceversa. The complete scheme of the adopted FSM is reported in Fig. 6. The time window of $1 \mathrm{~s}$ was experimentally selected after the repeated trials with patients and resulted in-line with the one proposed in [26].

A LED board is mounted on the support base of the device to give to the patients a visual feedback of the selected commands. In particular, a yellow LED blinks when a contraction of the muscle is recognized by the system. When flexion is selected an orange LED is turn on, while a green LED shows when extension is selected. Finally a red LED is turn on when the Soft-SixthFinger is stopped. 


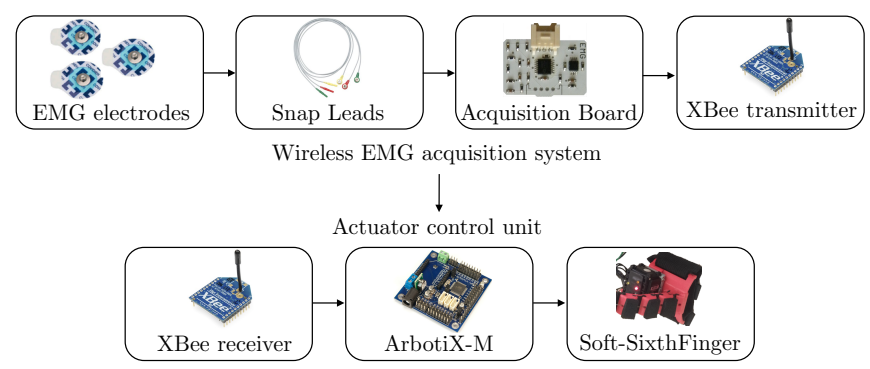

Fig. 5. The EMG wireless interface and actuator control unit. The acquired EMG data are transmitted wireless to the actuator's controller.

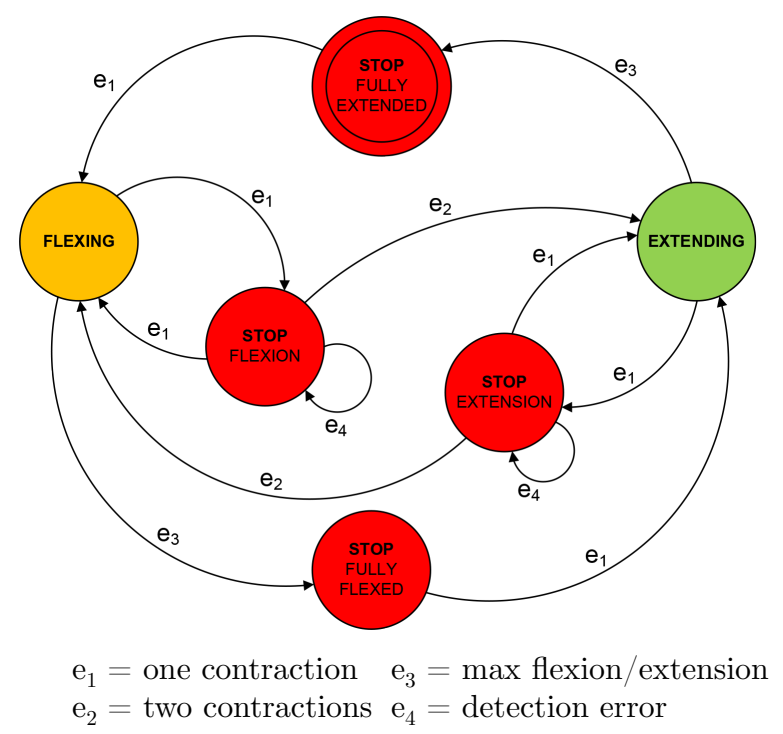

Fig. 6. The FSM proposed for the control of the Soft-SixthFinger. Events $e_{1}$ and $e_{2}$ are generated by the user, while events $e_{3}$ is generated by the software. The event $e_{4}$ can occur when, due to the noise in the system, more than two crosses of the threshold are registered in the time window.

\section{TESTS WITH CHRONIC PATIENTS}

\section{A. The Frenchay Arm Test}

Six chronic stroke patients (five male, one female, age 40-62) took part to the tests on how the Soft-SixthFinger can be used for hand grasping compensation. The proposed compensatory tool can be used by subjects showing a residual mobility of the arm. For being included in the experimental phase, patients had to score $\leq 2$ when their motor function was tested with the National Institute of Health Stroke Scale (NIHSS) [30], item 5 "paretic arm". Moreover, the patients had to show the following characteristics: normal consciousness (NIHSS, item 1a, 1b, 1c = 0), absence of conjugate eyes deviation (NIHSS, item $2=0$ ), absence of complete hemianopia (NIHSS, item $3 \leq 1$ ), absence of ataxia (NIHSS, item $7=0$ ), absence of completely sensory loss (NIHSS, item $8 \leq 1$ ), absence of aphasia (NIHSS, item $9=0$ ), absence of profound extinction and inattention (NIHSS, item $11 \leq 1$ ).

The goal of the tests was to verify how quickly the patients can learn to use the device and its EMG control interface. We performed a fully ecological qualitative test, the Frenchay Arm Test [16]. The test consisted of five tasks to be executed within three minutes:
1) Task 1: Stabilize a ruler, while drawing a line with a pencil held in the other hand. To pass, the ruler must be held firmly.

2) Task 2: Grasp a cylinder (12 mm diameter, $5 \mathrm{~cm}$ long), set on its side approximately $15 \mathrm{~cm}$ from the table edge, lift it about $30 \mathrm{~cm}$ and replace without dropping.

3) Task 3: Pick up a glass, half full of water positioned about 15 to $30 \mathrm{~cm}$ from the edge of the table, drink some water and replace without spilling ${ }^{2}$.

4) Task 4: Remove and replace a sprung clothes peg from a $10 \mathrm{~mm}$ diameter dowel, $15 \mathrm{~cm}$ long set in a $10 \mathrm{~cm}$ base, 15 to $30 \mathrm{~cm}$ from table edge. Not to drop peg or knock dowel over.

5) Task 5: Comb hair (or imitate); must comb across top, down the back and down each side of head.

The patient scored 1 for each of the successfully completed task, while he or she scored 0 in case of fail. The subject sat at a table with his hands in his lap, and each task started from this position. He or she was then asked to use his or her affected arm/hand to execute the tasks. Although the Frenchay arm test has not been specifically designed for evaluating compensatory tools, it has shown good reliability in measuring functional changes in stroke patients when comparing with other upper limb assessments [16]. The Frenchay Arm Test contains manipulation tasks of everyday life activity that evaluate the capability of grasping an object without a deep involvement of the patient arm. Other tests like, e.g., ARAT and UEFT [31], are more suitable for the evaluation of arm mobility which is out of the scope of this work. Patients wore the Soft-SixthFinger in their paretic limb, the left hand for two subjects and the right one for the other four. The number of modules on the device was chosen according to the selected tasks. Seven modules were used in order to grasp the proposed objects. Written informed consent was obtained from all participants. The procedures were in accordance with the Declaration of Helsinki.

The rehabilitation team assisted the subjects during a training phase that lasted for about one hour. During this phase, the optimal position of the device on the arm, according to the patient motor deficit, was evaluated. Moreover, the eCap parameters were tuned according to the patient.

After the training phase, the subjects had three minutes to perform the Frenchay Arm Test. Three patients tried the extra-finger for the first time. All the subjects performed the test two times, one without and one with the SoftSixthFinger. The starting condition was selected randomly. The results of the test are shown in Table III for the six patients. Screenshots of the tasks are reported in Fig. 7. Note that in the execution of Task 1 the Soft-SixthFinger do not interfere with the paretic limb action. The patients can stabilize the ruler without using any external help, so the device is kept in its rest position.

\section{B. Bimanual tasks}

The latter phase of post-stroke rehabilitation is based on the learning of newly acquired motor strategies to compensate the neurological deficit. These strategies may sometimes be neither ergonomic nor ecological, or may even

\footnotetext{
${ }^{2}$ Note that for safety reasons we did not use water in presence of electronic components.
} 
TABLE III

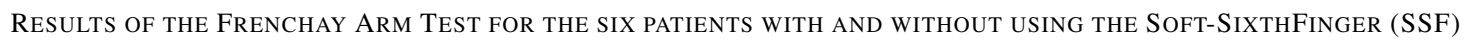

\begin{tabular}{|l|c|c|c|c|c|c|c|c|c|c|c|c|}
\hline \multirow{2}{*}{ Frenchay Arm Test } & \multicolumn{2}{|c|}{ Patient 1 } & \multicolumn{2}{c|}{ Patient 2 } & \multicolumn{2}{c|}{ Patient 3 } & \multicolumn{2}{c|}{ Patient 4 } & \multicolumn{2}{c|}{ Patient 5 } & \multicolumn{2}{c|}{ Patient 6} \\
& SSF & no SSF & SSF & no SSF & SSF & no SSF & SSF & no SSF & SSF & no SSF & SSF & no SSF \\
\hline Stabilize a ruler & 1 & 1 & 1 & 1 & 1 & 1 & 1 & 1 & 1 & 1 & 1 & 1 \\
\hline Grasp a cylinder & 1 & 0 & 1 & 0 & 1 & 0 & 1 & 0 & 1 & 0 & 1 & 0 \\
\hline Pick up a glass & 1 & 0 & 1 & 0 & 1 & 0 & 1 & 0 & 1 & 0 & 1 & 0 \\
\hline Remove a sprung & 0 & 0 & 0 & 0 & 0 & 0 & 0 & 0 & 0 & 0 & 0 & 0 \\
\hline Comb hair & 0 & 0 & 0 & 0 & 0 & 0 & 0 & 0 & 0 & 0 & 1 & 0 \\
\hline
\end{tabular}

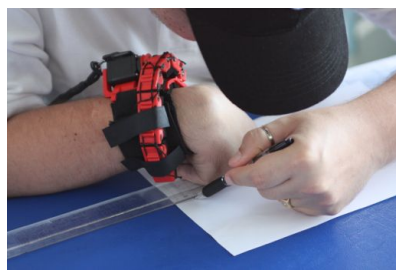

Task 1: Stabilize a ruler
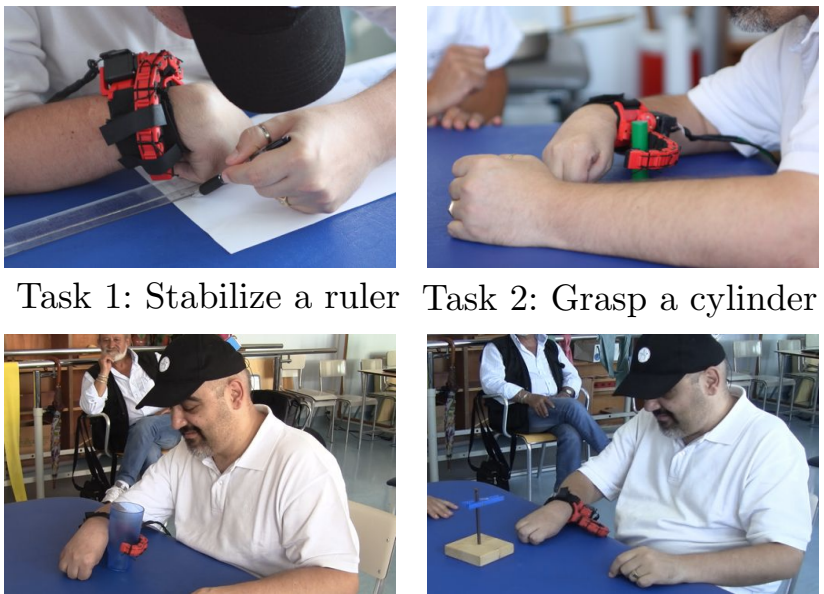

Task 2: Grasp a cylinder

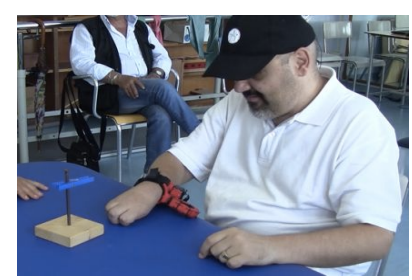

Task 3: Pick up a glass Task 4: Remove a sprung

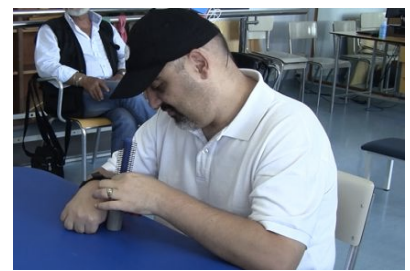

Task 5: Comb hair

Fig. 7. The five tasks of the Frenchay Arm Test.

increase pathological motor patterns, usually by worsening tonic flexion at the forearm of the paretic limb [32]. Some compensation techniques take also advantage of dedicated objects that allows the users to execute typical bimanual tasks only with the healthy hand. An example of available aids is reported in Fig. 8. However, the use of such tools is usually limited to the structured houses of the patients, restricting the possibilities of the patients to exploit them outside. The Soft-SixthFinger is a portable compensatory tool that can be carried as a bracelet when not used. This allows the patients to bring the devices wherever they want. The regained capability of grasping object with the help of the device, stimulate the patient to use his paretic limb so preserving residual mobility.

As a proof of concept, we tested the device in four different bimanual tasks typical of ADL. The four tasks were:

- unscrew the cap of a tomato jar;

- open a can of beans;

- open a squared can of cat food;

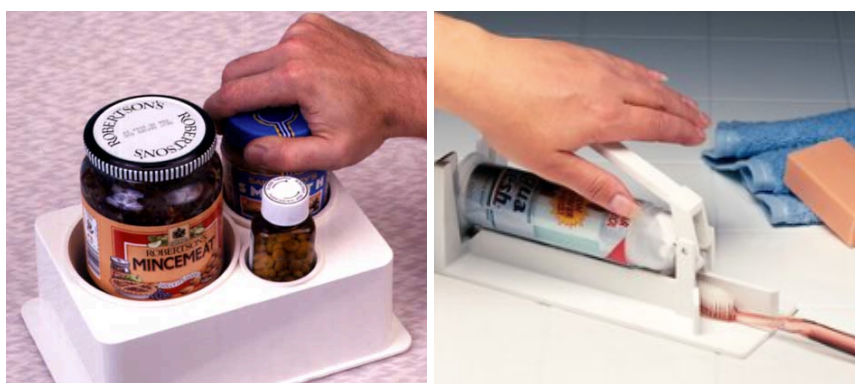

Fig. 8. Examples of adaptive kitchen aids designed to be used with only one hand. On the left, a case used as aid for bottle and jar opening. On the right, a toothpaste dispenser. Pictures courtesy of Elderstore ${ }^{\circledR}$
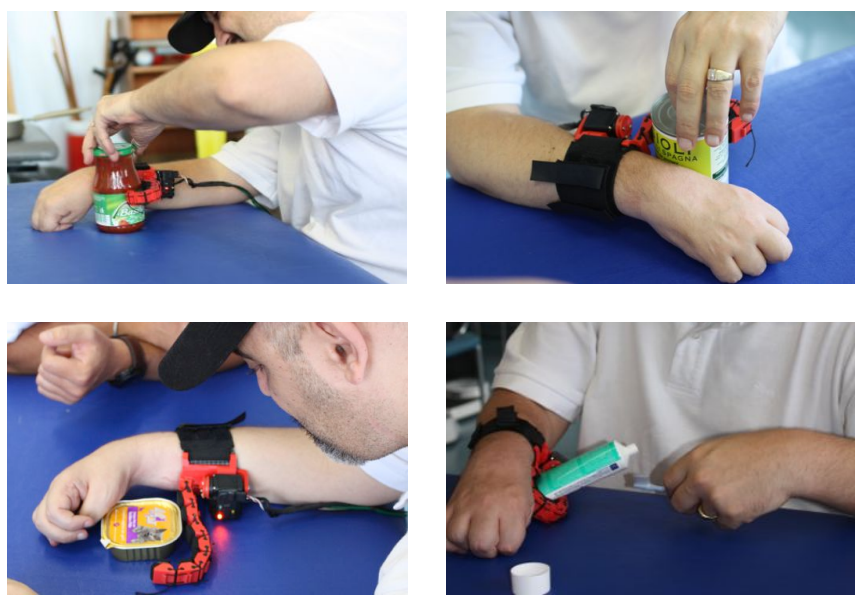

Fig. 9. The four tasks. From the top left, clockwise: unscrew a jar, open a can, squeeze a toothpaste tube and open a squared can.

- squeeze toothpaste tube over a toothbrush.

In all these bimanual tasks, the paretic limb and the SoftSixthFinger work together to constrain the motion of the object while the healthy hand manipulate it (e.g., constrain the motion of the tomato jar while the healthy hand is unscrewing its cap). Pictures of the execution of the four tasks are reported in Fig. 9. All the patients were able to execute the four tasks without requiring a specific training. Note that, thanks to the passive compliance embedded in its structure, the device was able to adapt also to squared shapes like the can of cat food.

\section{Questionnaire}

After the Frenchay Arm Test and the bimanual tasks, we investigated the users' subjective satisfaction and possible 
concerns related to the proposed system. According to [33], questionnaires and interviews are useful methods for studying how users use systems and what features they particularly like or dislike. The patients were asked to fill the UsefulnessSatisfaction-and-Ease-of-use-questionnaire (USE, [34]) that focuses on the experience of the system usage. This questionnaire uses a seven-point Likert rating scale. Mean and standard deviation (SD) of the questionnaire factors are presented in Table IV.

TABLE IV

QUESTIONNAIRE FACTORS AND RELATIVE MARKS. THE MARK RANGES FROM " 1 = STRONGLY DISAGREE" TO " $7=$ STRONGLY AGREE". MEAN AND STANDARD DEVIATION (MEAN (SD)) ARE REPORTED.

\begin{tabular}{|l|c|}
\hline Questionnaire factors & Mean (SD) \\
\hline \hline Usefulness & $4.6(0.8)$ \\
\hline Ease of use & $5.6(0.6)$ \\
\hline Ease of learning & $6.5(0.8)$ \\
\hline Satisfaction & $5.8(0.7)$ \\
\hline
\end{tabular}

\section{CONCLUSION}

The Soft-SixthFinger is the result of the synergistic effort of engineers, clinicians and a small group of patients wishing to improve their upper limb functionality. Patients suggestions on the possible uses of the device and on its positioning on the forearm has steered the design towards the solution presented in this work. The proposed underactuated and passively compliant device requires approximately one hour training before to be correctly used by newbies. Elastic joint and tendon driven actuation also resulted in a robust device against unwanted contact with the environment. Robustness is paramount in the grasp approaching phase since the reduced mobility of the paretic limb in the chronic stroke patients frequently leads to an heavy sliding of the device over the surface where the object is placed.

In the Soft-SixthFinger flexible parts are introduced in the kinematic structure to achieve passive compliance. Using compliant flexible joints and underactuation, we have designed a devices that can passively adapt to object size and shape. The adaptive joint coupling and transmission design lowers the necessity of complicated sensing and control through passive adaptation to the object shape.

Another important feature of the proposed device is its safety in the use. In fact, traditional exoskeletons need several precautions in terms of kinematics design to allow the human to feel no restriction to his or her natural motion patterns avoiding possible source of non-ergonomic interaction forces. The use of the extra-finger results to be safer in this regard since no coupling with the human limb joints is required.

The proposed questionnaire reveled that a simplification on the procedure of tuning the sensitivity of the EMG towards a self-tuning procedure is needed. On the other side, the users positively evaluated the wearability of the device.

We are currently investigating the possibility of using our device in patients affected by other neurological diseases possibly affecting hand grasping, such as Multiple Sclerosis,
Amyotrophic Lateral Sclerosis and paresis due to cervical spinal cord lesions.

\section{ACKNOWLEDGMENTS}

The authors are grateful to Dr. David Cioncoloni, Dr. Emiliano Santernecchi, Sabrina Taddei and Prof. Simone Rossi for their help in recruiting patients and assist them during the tests.

\section{REFERENCES}

[1] A. S. Go, D. Mozaffarian, V. L. Roger, E. J. Benjamin, J. D. Berry, M. J. Blaha, S. Dai, E. S. Ford, C. S. Fox, S. Franco, et al., "Heart disease and stroke statistics-2014 update: a report from the american heart association.," Circulation, vol. 129, no. 3, p. e28, 2014.

[2] A. Sunderland, D. Fletcher, L. Bradley, D. Tinson, R. L. Hewer, and D. T. Wade, "Enhanced physical therapy for arm function after stroke: a one year follow up study.," Journal of Neurology, Neurosurgery \& Psychiatry, vol. 57, no. 7, pp. 856-858, 1994.

[3] G. Kwakkel, B. J. Kollen, J. van der Grond, and A. J. Prevo, "Probability of regaining dexterity in the flaccid upper limb impact of severity of paresis and time since onset in acute stroke," Stroke. vol. 34, no. 9, pp. 2181-2186, 2003

[4] A. C. Lo, P. D. Guarino, L. G. Richards, J. K. Haselkorn, G. F Wittenberg, D. G. Federman, R. J. Ringer, T. H. Wagner, H. I. Krebs, B. T. Volpe, et al., "Robot-assisted therapy for long-term upper-limb impairment after stroke," New England Journal of Medicine, vol. 362 no. 19, pp. 1772-1783, 2010.

[5] G. Kwakkel, B. J. Kollen, and H. I. Krebs, "Effects of robot-assisted therapy on upper limb recovery after stroke: a systematic review," Neurorehabilitation and neural repair, 2007.

[6] Y. Hasegawa, Y. Mikami, K. Watanabe, Z. Firouzimehr, and Y. Sankai, "Wearable handling support system for paralyzed patient," in Intelligent Robots and Systems, 2008. IROS 2008. IEEE/RSJ International Conference on, pp. 741-746, IEEE, 2008.

[7] M. Iwaki, Y. Hasegawa, and Y. Sankai, "Study on wearable system for daily life support using mckibben pneumatic artificial muscle," in Intelligent Robots and Systems (IROS), 2010 IEEE/RSJ International Conference on, pp. 3670-3675, IEEE, 2010.

[8] J. L. Pons et al., Wearable robots: biomechatronic exoskeletons, vol. 338. Wiley Online Library, 2008.

[9] D. Prattichizzo, M. Malvezzi, I. Hussain, and G. Salvietti, "The sixthfinger: a modular extra-finger to enhance human hand capabilities," in Proc. IEEE Int. Symp. in Robot and Human Interactive Communication, (Edinburgh, United Kingdom), 2014.

[10] I. Hussain, L. Meli, C. Pacchierotti, G. Salvietti, and D. Prattichizzo, "Vibrotactile haptic fedback for intuitive control of robotic extra fingers," in Proc. IEEE World Haptics Conference (WHC), (Chicago, IL), 2015

[11] I. Hussain, G. Salvietti, L. Meli, C. Pacchierotti, and D. Prattichizzo, "Using the robotic sixth finger and vibrotactile feedback for grasp compensation in chronic stroke patients," in Proc. IEEE/RAS-EMBS International Conference on Rehabilitation Robotics (ICORR), 2015.

[12] L. U. Odhner, L. P. Jentoft, M. R. Claffee, N. Corson, Y. Tenzer, R. R. Ma, M. Buehler, R. Kohout, R. D. Howe, and A. M. Dollar, "A compliant, underactuated hand for robust manipulation," The International Journal of Robotics Research, vol. 33, no. 5, pp. 736-752, 2014.

[13] R. Deimel and O. Brock, "A novel type of compliant, underactuated robotic hand for dexterous grasping," Robotics: Science and Systems, Berkeley, CA, pp. 1687-1692, 2014

[14] M. G. Catalano, G. Grioli, E. Farnioli, A. Serio, C. Piazza, and A. Bicchi, "Adaptive synergies for the design and control of the pisa/iit softhand," The International Journal of Robotics Research, vol. 33 no. 5, pp. 768-782, 2014.

[15] M. Grebenstein, M. Chalon, W. Friedl, S. Haddadin, T. Wimböck, G. Hirzinger, and R. Siegwart, "The hand of the dlr hand arm system: Designed for interaction," The International Journal of Robotics Research, vol. 31, no. 13, pp. 1531-1555, 2012.

[16] A. Heller, D. Wade, V. A. Wood, A. Sunderland, R. L. Hewer and E. Ward, "Arm function after stroke: measurement and recovery over the first three months.," Journal of Neurology, Neurosurgery \& Psychiatry, vol. 50, no. 6, pp. 714-719, 1987.

[17] L. Birglen, T. Lalibertè, and C. Gosselin, Underactuated Robotic Hands, vol. 40 of Springer Tracts in Advanced Robotics. Springer, 2008

[18] A. M. Dollar and R. D. Howe, "The highly adaptive sdm hand: Design and performance evaluation," The international journal of robotics research, vol. 29, no. 5, pp. 585-597, 2010. 
[19] C. Eppner and O. Brock, "Grasping unknown objects by exploiting shape adaptability and environmental constraints," in Intelligent Robots and Systems (IROS), 2013 IEEE/RSJ International Conference on, pp. 4000-4006, Nov 2013.

[20] Robotis, "Dynamixel ax-12a robot actuator." On-line: http://www.trossenrobotics.com/dynamixel-ax-12-robot-actuator.aspx.

[21] ArbotiX, "Arbotix-m robocontroller,open source." On-line: http://www.trossenrobotics.com/p/arbotix-robot-controller.aspx.

[22] S. Bitzer and P. van der Smagt, "Learning emg control of a robotic hand: towards active prostheses," in Robotics and Automation, 2006. ICRA 2006. Proceedings 2006 IEEE International Conference on, pp. 2819-2823, IEEE, 2006.

[23] M. Zecca, S. Micera, M. Carrozza, and P. Dario, "Control of multifunctional prosthetic hands by processing the electromyographic signal," Critical Reviews in Biomedical Engineering, vol. 30, no. 4-6, 2002.

[24] K. Kiguchi, T. Tanaka, and T. Fukuda, "Neuro-fuzzy control of a robotic exoskeleton with emg signals," Fuzzy Systems, IEEE Transactions on, vol. 12, no. 4, pp. 481-490, 2004.

[25] M. DiCicco, L. Lucas, and Y. Matsuoka, "Comparison of control strategies for an emg controlled orthotic exoskeleton for the hand," in Robotics and Automation, 2004. Proceedings. ICRA'04. 2004 IEEE International Conference on, vol. 2, pp. 1622-1627, IEEE, 2004.

[26] T. Felzer and B. Freisleben, "Hawcos: the hands-free wheelchair control system," in Proceedings of the fifth international ACM conference on Assistive technologies, pp. 127-134, ACM, 2002.

[27] R. Merletti, A. Botter, A. Troiano, E. Merlo, and M. A. Minetto, "Technology and instrumentation for detection and conditioning of the surface electromyographic signal: state of the art," Clinical Biomechanics, vol. 24, no. 2, pp. 122-134, 2009.

[28] J. C. Huhta and J. G. Webster, "60-hz interference in electrocardiography," Biomedical Engineering, IEEE Transactions on, no. 2, pp. 91101, 1973.

[29] M. A. Oskoei and H. Hu, "Myoelectric control systems - a survey," Biomedical Signal Processing and Control, vol. 2, no. 4, pp. 275-294, 2007.

[30] T. Brott, H. Adams, C. P. Olinger, J. R. Marler, W. G. Barsan, J. Biller, J. Spilker, R. Holleran, R. Eberle, and V. Hertzberg, "Measurements of acute cerebral infarction: a clinical examination scale.," Stroke, vol. 20, no. 7, pp. 864-870, 1989.

[31] T. Platz, C. Pinkowski, F. van Wijck, I.-H. Kim, P. Di Bella, and G. Johnson, "Reliability and validity of arm function assessment with standardized guidelines for the fugl-meyer test, action research arm test and box and block test: a multicentre study," Clinical Rehabilitation, vol. 19, no. 4, pp. 404-411, 2005.

[32] S. L. Wolf, D. E. Lecraw, L. A. Barton, and B. B. Jann, "Forced use of hemiplegic upper extremities to reverse the effect of learned nonuse among chronic stroke and head-injured patients," Experimental neurology, vol. 104, no. 2, pp. 125-132, 1989.

[33] J. Nielsen, Usability engineering. Elsevier, 1994.

[34] A. M. Lund, "Measuring usability with the use questionnaire," Usability interface, vol. 8, no. 2, pp. 3-6, 2001. 